\title{
Effectiveness of Smartwatch Guidance for High-Quality Infant Cardiopulmonary Resuscitation: A Simulation Study
}

\author{
Seong A Jeon ${ }^{1}$, Hansol Chang ${ }^{1,2}$, Sun Young Yoon ${ }^{2}$, Nayeong Hwang ${ }^{3}$, Kyunga Kim ${ }^{3}$, Hee Yoon ${ }^{1}$, \\ Sung Yeon Hwang ${ }^{1}{ }^{\infty}$, Tae Gun Shin ${ }^{1}{ }^{(}$, Won Chul Cha ${ }^{1,2,4}$ and Taerim Kim ${ }^{1, *} \mathbb{E}$ \\ 1 Department of Emergency Medicine, Samsung Medical Center, Sungkyunkwan University School \\ of Medicine, Seoul 06351, Korea; sarahjun@naver.com (S.A.J.); hansol.chang@samsung.com (H.C.); \\ wildhi.yoon@samsung.com (H.Y.); sygood.hwang@samsung.com (S.Y.H.); \\ taegun.shin@samsung.com (T.G.S.); wc.cha@samsung.com (W.C.C.) \\ 2 Department of Digital Health, Samsung Advanced Institute for Health Sciences and Technology, \\ Sungkyunkwan University, Seoul 06355, Korea; roseherb21@naver.com \\ 3 Biostatistics and Clinical Epidemiology Center, Samsung Medical Center, Seoul 06351, Korea; \\ ny.hwang@snri.co.kr (N.H.); Kyunga.j.kim@samsung.com (K.K.) \\ 4 Health Information and Strategy Center, Samsung Medical Center, 81 Irwon-ro Gangnam-gu, \\ Seoul 06351, Korea \\ * Correspondence: taerim.j.kim@gmail.com; Tel.: +82-2-3410-2053; Fax: +82-2-3410-0049
}

check for updates

Citation: Jeon, S.A.; Chang, H.; Yoon, S.Y.; Hwang, N.; Kim, K.; Yoon, H.; Hwang, S.Y.; Shin, T.G.; Cha, W.C.; Kim, T. Effectiveness of Smartwatch Guidance for High-Quality Infant Cardiopulmonary Resuscitation: A Simulation Study. Medicina 2021, 57, 193. https://doi.org/10.3390/ medicina57030193

Academic Editor: Wilhelm Behringer

Received: 3 February 2021

Accepted: 22 February 2021

Published: 25 February 2021

Publisher's Note: MDPI stays neutral with regard to jurisdictional claims in published maps and institutional affiliations.

Copyright: (c) 2021 by the authors. Licensee MDPI, Basel, Switzerland. This article is an open access article distributed under the terms and conditions of the Creative Commons Attribution (CC BY) license (https:// creativecommons.org/licenses/by/ $4.0 /)$.

\begin{abstract}
Background and objectives: As in adults, the survival rates and neurological outcomes after infant Cardiopulmonary resuscitation (CPR) are closely related to the quality of resuscitation. This study aimed to demonstrate that using a smartwatch as a haptic feedback device increases the quality of infant CPR performed by medical professionals. Materials and methods: We designed a prospective, randomized, case-crossover simulation study. The participants $(\mathrm{n}=36)$ were randomly allocated to two groups: control first group and smartwatch first group. Each CPR session consisted of $2 \mathrm{~min}$ of chest compressions (CCs) using the two-finger technique (TFT), $2 \mathrm{~min}$ of rest, and $2 \mathrm{~min}$ of CCs using the two-thumb encircling hands technique (TTHT). Results: The primary outcome was the variation in the "proportion of optimal chest compression duration" and "compression rate" between the smartwatch-assisted and non-smartwatch-assisted groups. The secondary outcome was the variation in the "compression depth" between two groups. The proportion of optimal CC duration was significantly higher in the smartwatch-assisted group than in the non-smartwatch-assisted group. The absolute difference from 220 was much smaller in the smartwatch-assisted group (218.02) than in the non-smartwatch-assisted group (226.59) ( $p$-Value $=0.018)$. Conclusion: This study demonstrated the haptic feedback system using a smartwatch improves the quality of infant CPR by maintaining proper speed and depth regardless of the compression method used.
\end{abstract}

Keywords: wearable electronic devices; cardiopulmonary resuscitation; heart arrest; infant; simulation training; feedback

\section{Introduction}

While the incidence of pediatric out-of-cardiac-arrest (OHCA) is low, that of infant OHCA is 10-fold higher, approaching the incidence in adults [1]. In addition, the survival rates for infants receiving cardiopulmonary resuscitation (CPR) are reported to be twice as poor as those for other pediatric age groups [1]. Previous studies have shown that neither the incidence nor the survival rates of pediatric OHCA have improved over the last decades [2]. As in adults, the survival rates and neurological outcomes after infant CPR are closely related to the quality of resuscitation [3-5]. Minimizing chest compression interruptions and allowing full chest recoil after each compression are the basic processes of the high-quality CPR [6,7].

According to the 2018 American Heart Association (AHA) guidelines for infant CPR, the compression depth should be at least one-third of the diameter of the front and back of 
the chest, which is approximately 1.5 inches $(4 \mathrm{~cm})$ [8]. The recommended compression rate is between 100 and 120 times per minute [9-11]. However, as stated in the guidelines, it is not easy to maintain good-quality chest compression (CC) in the field [12]. Therefore, various types of feedback devices have been developed to improve the quality of chest CCs [13-15].

Most of the devices are visual and auditory devices that indicated the depth, speed, and appropriateness of CCs [12]. However, the audio-visual feedback devices are difficult for users to focus on and use in crowded and noisy CPR environments [16]. In addition, these devices often need to be attached to the chest wall of patients and are designed for adults, so their use in infant CPR is limited as the CCs are carried out with two fingers [17]. Currently, wearable devices have been developed for healthcare workers, providing feedback through a smartwatch [18]. Haptic devices using vibrations from a smartwatch can be applied more effectively than audio-visual feedback devices in the field $[17,19,20]$.

Although previous studies have shown that a smartwatch with real-time feedback can improve CPR quality, most were limited to adult CPR [21]. Furthermore, previous studies on infant CPR have focused on haptic feedback and medical students [17]. This study aimed to determine the effectiveness of smartwatch feedback in the delivery of high-quality infant CPR by medical professionals.

\section{Materials and Methods}

\subsection{Study Design}

This was a prospective, randomized, case-crossover simulation study that aimed to demonstrate the effect of a smartwatch-type haptic device on the quality of CC in infant CPR. We conducted a randomized, controlled mannequin-simulation study of a single rescuer hands-only CPR at the ED of Samsung Medical Center from 1 to 31 December 2019. The study was approved by the institutional review board of Samsung Medical Center (No. 2019-07-042-007) on 2 December 2019. All procedures were carried out in accordance with the Declaration of Helsinki.

\subsection{Study Participants}

Based on previous studies on adult CPR with haptic devices, we calculated our sample size. A size of 36 participants per group was calculated using the McNemar test for two paired proportions, with 0.025 significance and 0.80 power. The proportion of discordant pairs was assumed to be $50 \%$; based on that, the proportion of optimal duration of CCs using a smartwatch was $95 \%$ and that of CCs without using a smartwatch was $45 \%$. We hypothesized that there is no difference in the optimal duration between the two-finger technique (TFT) and the two-thumb encircling hands technique (TTHT).

Participants were recruited via recruitment information on the employee notice board. Medical professionals such as doctors, nurses, and emergency medical technicians (EMTs) who were either Pediatric Advanced Life Support (PALS), Basic Life Support (BLS), or Advanced Cardiovascular Life Support (ACLS) certified or had previous experience with infant CPR were eligible for participation. Volunteers were excluded if they had cardiovascular or musculoskeletal disease. Informed consent was attained from all the participants.

\subsection{Study Protocol}

All study participants were provided an introduction to the study protocol and the optimal cardiac compression for CPR suggested by the AHA guidelines that lasted for $10 \mathrm{~min}$. At the first visit, the participants were randomly allocated to two groups: control first group (Group A, $n=18$ ) and the smartwatch first group (Group B, $n=18$ ). Each session consisted of $2 \mathrm{~min}$ of CCs using TFT, 2 min of rest, and $2 \mathrm{~min}$ of CCs using the TTHT. All participants performed hands-only $\mathrm{CPR}$ according to the AHA guideline.

Group A performed two sets of CC (2 min each) without the feedback of the smartwatch at the first visit. CC sets comprised $2 \mathrm{~min}$ of TFT and $2 \mathrm{~min}$ of TTHT. The smartwatch 
first group (Group B) performed CCs while wearing a smartwatch with the preinstalled app at the first visit. The participants were then crossed over to the other group and performed CCs after a washout period of more than $6 \mathrm{~h}$. The study process is illustrated in Figure 1. A rate of $100-120 \mathrm{CCs} / \mathrm{min}$ was considered the optimal rate. The total number of CCs was measured during the $2 \mathrm{~min}$, and 0.5 to $0.6 \mathrm{~s}$ per one CC was considered the optimal duration.

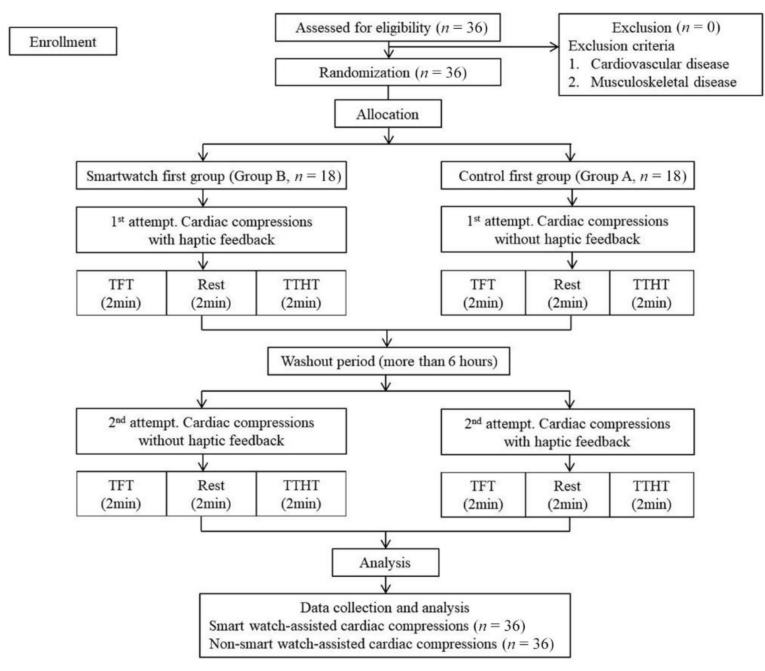

Figure 1. CONSORT flow diagram of the study. TFT, two-finger technique; TTHT, two-thumb encircling hands technique; $\mathrm{min}$, minute.

\subsection{Description of the Devices}

"Laerdal ${ }^{\circledR}$ Resusci baby" was used to gather and store the CC performance data in a laptop. Chest compressions are performed on the 'Resusci baby' mannequin and the number and depth of chest compressions performed by the subject are set to be recorded in the program linked to the mannequin. The "Samsung ${ }^{\circledR}$ Galaxy Gear S3" smartwatch (Samsung Electronics Inc., Seoul, Republic of Korea) with a haptic metronome application (on the Galaxy Store app called Wearable Metronome) was used in this study. This smartwatch was to be worn around the wrist and was set to provide constant vibrations at the rate of $110 / \mathrm{min}$ (Figure 2).
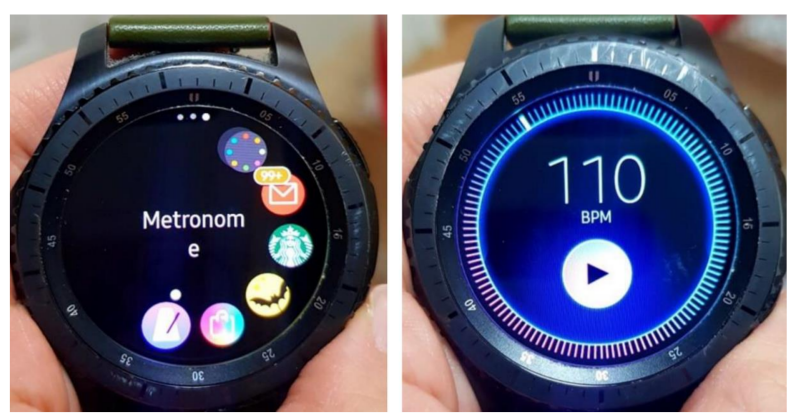

Figure 2. The smartwatch application is set to 110 BPM. BPM, beats per minute.

\subsection{Measures}

We gathered demographic information such as age, sex, job, and infant CPR experience from each subject. For outcome measures, the number of CCs during $2 \mathrm{~min}$, chest compression duration, and compression depth per stroke were collected. Thirty-second intervals were defined as quartiles in a sequence: first quartile (1Q), second quartile (2Q), third quartile $(3 \mathrm{Q})$, and fourth quartile $(4 \mathrm{Q})$. 


\subsection{Outcomes}

The primary outcome was the variation in the "proportion of optimal chest compression duration" and "compression rate". We compared the compression rate between the two groups. The secondary outcome was the variation in the "compression depth" between two groups. Subgroup analyses were further conducted to investigate the effect of smartwatch guidance for each compression technique, i.e., TFT and TTHT, and for every 30-s quartile.

\subsection{Data Analysis}

The demographic characteristics of each group were analyzed. Continuous variables with normal distribution were presented as mean (standard deviation (SD)) and those with non-normal distribution, as medians with interquartile rages (IQRs). Categorical variables were presented as counts and percentages. Two-sample $t$-test was used to analyze the data of two continuous variables with normal distribution, and Mann-Whitney U test was used to analyze the data of variables that were not normally distributed. Chi-square test was used to compare the frequencies of categorical variables between two groups.

We used the generalized estimation equation (GEE) models and odds ratio (ORs) with $95 \%$ confidence intervals to analyze repeatedly measured variables during multiple time points. In the GEE models, the measured variables were the CC duration and depth. The use of smartwatch and the type of compression technique were included as explanatory variables, and the interaction effect was also analyzed along with their primary effects. Subgroup analyses were further carried out for each technique. Statistical significance was set at $95 \%$ level ( $p$-Value of $<0.05$ ). Statistical analysis was performed using Statistical Analysis System (SAS) version 9.4 (SAS Institute, Cary, NC, USA) and R 3.5.1 (R Foundation for Statistical Computing).

\section{Results}

In this study, 36 participants were recruited and randomly allocated to two groups. Of them, 28 were physicians, 6 were paramedics, and 2 were nurses. None of the participants dropped out. Table 1 shows the demographic characteristics of the study population. The smartwatch first group showed less experience of CC within 1 month (smartwatch first group: $2.0(0.0-5.0)$ vs. $4.0(3.0-5.0), p=0.032)$; otherwise, there were no significant differences between the two groups in terms of age, sex, job, work experience, and experience of CC within 5 years.

Table 1. Baseline characteristics between smartwatch first group and control first group.

\begin{tabular}{|c|c|c|c|}
\hline & $\begin{array}{l}\text { Smartwatch } \\
\text { First Group } \\
\quad(n=18)\end{array}$ & $\begin{array}{l}\text { Control } \\
\text { First Group } \\
\quad(n=18)\end{array}$ & $p$-Value \\
\hline Age, median (IQR) & $28.5(25.0-30.0)$ & $27.5(25.0-31.0)$ & 0.622 \\
\hline Sex, $n(\%)$ & & & 1 \\
\hline Male & $6(33.3 \%)$ & $7(38.9 \%)$ & \\
\hline Female & $12(66.7 \%)$ & $11(61.1 \%)$ & \\
\hline Job, $n(\%)$ & & & 0.298 \\
\hline Doctor & $12(66.6 \%)$ & $16(88.8 \%)$ & \\
\hline EMT & $5(27.8 \%)$ & $1(5.6 \%)$ & \\
\hline Nurse & $1(5.6 \%)$ & $1(5.6 \%)$ & \\
\hline Work experience, $n(\%)$ & & & 1 \\
\hline Up to 3 years & $9(50.0 \%)$ & $8(44.4 \%)$ & \\
\hline More than 3 years & $9(50.0 \%)$ & $10(55.6 \%)$ & \\
\hline Experience of cardiac compression within 5 years (IQR) & $30.0(10.0-50.0)$ & $30.0(20.0-50.0)$ & 0.662 \\
\hline Experience of cardiac compression within 1 month (IQR) & $2.0(0.0-5.0)$ & $4.0(3.0-5.0]$ & 0.032 \\
\hline
\end{tabular}

Values are presented as median with IQR or number (proportion). 


\subsection{Result for CC Performance}

The CC performance results are shown in Table 2. The proportion of optimal CC duration was significantly different between the smartwatch-assisted CCs and non-smartwatchassisted CCs. Both groups achieved satisfactory mean CC duration of $0.5-0.6 \mathrm{~s}$ (smartwatchassisted CCs (550.8 $\pm 54.8 \mathrm{msec})$ vs. non-smartwatch-assisted CCs $(529.9 \pm 68.4 \mathrm{msec})$, $p$-Value $<0.001)$. However, the proportion of optimal CC duration was higher in the smartwatch-assisted CCs (smartwatch-assisted CCs $(11,081 / 71.1 \%)$ vs. non-smartwatchassisted CCs $(9,630 / 59.4 \%), p$-Value $<0.001)$.

Table 2. Proportion of optimal CC duration between smartwatch-assisted and non-smartwatchassisted groups.

\begin{tabular}{cccc}
\hline & $\begin{array}{c}\text { Smartwatch } \\
\text {-Assisted CCs }\end{array}$ & $\begin{array}{c}\text { Non-Smartwatch } \\
\text {-Assisted CCs }\end{array}$ & $\boldsymbol{p}$-Value \\
\hline Total number of chest compression & 15,585 & 16,212 & \\
Proportion of optimal chest compression (\%) & $11,081(71.1 \%)$ & $9630(59.4 \%)$ & $<0.001$ \\
Chest compression duration (msec) & $550.8 \pm 54.8$ & $529.9 \pm 68.4$ & $<0.001$ \\
\hline CC, chest compression; msec, millisecond. The values are presented as mean \pm standard deviation or number (\%).
\end{tabular}

The results of subgroup analysis for compression duration between the groups are shown in Table 3. The average duration of smartwatch-assisted CCs was about $18 \mathrm{~ms}$ longer than that of non-smartwatch-assisted CCs, with statistical significance $(p<0.001)$. The estimates for 2Q, 3Q, and 4Q were, respectively, 9.39 (95\% CI, 4.69-14.09), 12.95 (6.78-19.13), and 13.69 (7.44-19.93) seconds longer than the estimate for Q1, with statistical significance $(p<0.001)$. We conducted interaction analyses between with or without smartwatchassisted CCs and four interquartiles. The results showed that the effect of smartwatchassisted CCs was insignificantly different along the four interquartiles. Smartwatch-assisted CCs showed generally increasing quality from $1 \mathrm{Q}$ to $4 \mathrm{Q}$. In another subgroup analysis between TFT and TTHT, the CC duration did not show a significant difference.

Table 3. Subgroup analysis for compression duration between smartwatch-assisted and non-smartwatch-assisted groups.

\begin{tabular}{|c|c|c|c|c|c|c|c|c|}
\hline Parameter & \multicolumn{2}{|l|}{ Level (vs. Ref.) } & Estimate & $\begin{array}{l}\text { Standard } \\
\text { Error }\end{array}$ & \multicolumn{2}{|c|}{ 95\% Confidence Limits } & $\mathbf{Z}$ & $p$-Value \\
\hline \multirow[t]{2}{*}{ Group } & \multicolumn{2}{|c|}{$\begin{array}{c}\text { Smartwatch-assisted CCs } \\
\text { vs. Non-smartwatch-assisted CCs }\end{array}$} & 18.15 & 4.79 & 8.75 & 27.54 & 3.78 & $<0.001$ \\
\hline & \multicolumn{2}{|l|}{$2 \mathrm{Q}$ vs. $1 \mathrm{Q}$} & 9.39 & 2.40 & 4.69 & 14.09 & 3.92 & \\
\hline \multirow[t]{2}{*}{ Quartile } & \multicolumn{2}{|l|}{$3 \mathrm{Q}$ vs. $1 \mathrm{Q}$} & 12.95 & 3.15 & 6.78 & 19.13 & 4.11 & $<0.001$ \\
\hline & \multicolumn{2}{|l|}{$4 \mathrm{Q}$ vs. 1Q } & 13.69 & 3.19 & 7.44 & 19.93 & 4.3 & \\
\hline \multirow[t]{2}{*}{ Method } & \multicolumn{2}{|l|}{ TTHT vs. TFT } & -0.32 & 4.53 & -9.19 & 8.55 & -0.07 & 0.943 \\
\hline & \multirow{3}{*}{$\begin{array}{l}\text { Smartwatch-assisted CCs vs. } \\
\text { Non-smartwatch-assisted CCs }\end{array}$} & $2 Q$ vs. $1 Q$ & 0.86 & 2.40 & -3.85 & 5.57 & 0.36 & \\
\hline \multirow{2}{*}{$\begin{array}{l}\text { Group* } \\
\text { quartile }\end{array}$} & & $3 \mathrm{Q}$ vs. $1 \mathrm{Q}$ & 3.99 & 3.50 & -2.86 & 10.84 & 1.14 & 0.266 \\
\hline & & $4 Q$ vs. $1 Q$ & 6.16 & 3.44 & -0.58 & 12.89 & 1.79 & \\
\hline
\end{tabular}

TFT, two-finger technique; TTHT, two-thumb encircling hands technique; $Q$, quartile; $1 \mathrm{Q}$, first quartile; $2 \mathrm{Q}$, second quartile; $3 \mathrm{Q}$, third quartile; $4 \mathrm{Q}$, Fourth quartile.

We compared the compression rate between the smartwatch-assisted CCs and nonsmartwatch-assisted CCs. The total number of CCs was counted for $2 \mathrm{~min}$. We set the average value of $110 / \mathrm{min}$ as the adequate speed for the smartwatch metronome and 220 CCs in $2 \mathrm{~min}$ as the appropriate rate. Table 4 shows that the compression rate of the smartwatch-assisted group was 8.56 times lower than that of the non-smartwatchassisted group, with statistical significance $(p=0.018)$. In the subgroup analysis between TFT and TTHT, the compression rate did not show a significant difference. The absolute difference from 220 was much smaller for the smartwatch assisted CCs (1.98) than for non-smartwatch-assisted CCs (6.59). The results are shown in Table 5. 
Table 4. Comparison of compression rate between smartwatch-assisted and non-smartwatch-assisted groups and TFT vs. TTHT group.

\begin{tabular}{|c|c|c|c|c|c|c|c|}
\hline Parameter & Level (vs. Ref.) & Estimate & Standard Error & \multicolumn{2}{|c|}{ 95\% Confidence Limits } & $\mathbf{Z}$ & $p$-Value \\
\hline Group & $\begin{array}{l}\text { Smartwatch-assisted CCs vs. } \\
\text { Non-smartwatch-assisted CCs }\end{array}$ & -8.56 & 3.63 & -15.68 & -1.45 & -2.36 & 0.018 \\
\hline Method & TTHT vs. TFT & 4.03 & 2.11 & -0.11 & 8.16 & 1.91 & 0.057 \\
\hline
\end{tabular}

CC, chest compression; TFT, two-finger technique; TTHT, two-thumb encircling hands technique.

Table 5. Absolute difference in compression rate from set value (220 times).

\begin{tabular}{cccc}
\hline Average Compressions & $\begin{array}{c}\text { Smartwatch } \\
\text {-Assisted CCs }\end{array}$ & $\begin{array}{c}\text { Non-Smartwatch } \\
\text {-Assisted CCs }\end{array}$ & Overall \\
\hline TFT & 216.01 & 224.57 & 220.29 \\
TTHT & 220.04 & 228.60 & 224.32 \\
Overall & 218.02 & 226.59 & 222.31 \\
Absolute difference from 220 & 1.98 & 6.59 & \\
\hline
\end{tabular}

$\overline{\mathrm{CC}}$, chest compression; TFT, two-finger technique; TTHT, two-thumb encircling hands technique. Values are presented as mean.

\subsection{Results for Compression Depth}

The mean CC depth was not significantly different between the smartwatch-assisted and non-smartwatch-assisted groups. The subgroup analysis showed that the adjusted odds ratios (AORs) for $2 \mathrm{Q}, 3 \mathrm{Q}$, and $4 \mathrm{Q}$ in comparison with $1 \mathrm{Q}$ for the compression depth were 0.58 (95\% CI, 0.43-0.79), 0.49 (0.34-0.72), and 0.40 (0.27-0.59), respectively (Table 6). The interaction analysis shows the AOR of the smartwatch-assisted CCs and its positive impact. The AORs for 2Q, 3Q, and 4Q in comparison with $1 \mathrm{Q}$ were 1.49 (95\% CI, 1.03-2.14), $1.80(1.13-2.87)$, and $2.16(1.33-3.52)$, respectively (Table 6). The appropriateness of the compression depth had gradually increased from $1 Q$ to $4 Q$ in smartwatch-assisted CCs (Figure 3).

Table 6. Subgroup analysis for compression depth between smartwatch-assisted and non-smartwatch-assisted groups.

\begin{tabular}{|c|c|c|c|c|c|c|c|c|c|c|c|}
\hline \multirow{2}{*}{$\begin{array}{c}\text { Parameter } \\
\text { Group }\end{array}$} & \multicolumn{2}{|l|}{ Level (vs. Ref.) } & \multirow{2}{*}{$\begin{array}{c}\text { Estimate } \\
-0.44\end{array}$} & \multirow{2}{*}{$\begin{array}{c}\begin{array}{c}\text { Standard } \\
\text { Error }\end{array} \\
0.27\end{array}$} & \multicolumn{2}{|c|}{ 95\% Confidence Limits } & \multirow{2}{*}{$\begin{array}{c}\mathbf{Z} \\
-1.67\end{array}$} & \multirow{2}{*}{$\begin{array}{c}p \text {-Value } \\
0.096\end{array}$} & \multirow{2}{*}{$\begin{array}{c}\begin{array}{c}\text { Odds } \\
\text { Ratio }\end{array} \\
0.64\end{array}$} & \multicolumn{2}{|c|}{$95 \%$ CI of OR } \\
\hline & $\begin{array}{l}\text { Smartwatch-assisted C } \\
\text { Non-smartwatch assiste }\end{array}$ & $\begin{array}{l}\text { S vs. } \\
\text { CCs }\end{array}$ & & & -0.96 & 0.08 & & & & 0.38 & 1.08 \\
\hline \multirow{3}{*}{ Quartile } & $2 \mathrm{Q}$ vs. $1 \mathrm{Q}$ & & -0.54 & 0.16 & -0.85 & -0.24 & -3.48 & \multirow{3}{*}{$<0.001$} & 0.58 & 0.43 & 0.79 \\
\hline & 3Q vs. 10 & & -0.71 & 0.19 & -1.09 & -0.34 & -3.71 & & 0.49 & 0.34 & 0.72 \\
\hline & $4 \hat{\mathrm{Q}}$ vs. $1 \hat{\mathrm{Q}}$ & & -0.91 & 0.20 & -1.30 & -0.52 & -4.56 & & 0.40 & 0.27 & 0.59 \\
\hline \multirow{4}{*}{$\begin{array}{l}\text { Method } \\
\text { Group* } \\
\text { Quartile }\end{array}$} & TTHT vs. TFT & & 2.14 & 0.33 & 1.49 & 2.78 & 6.49 & \multirow[t]{2}{*}{$<0.001$} & 8.49 & 4.45 & 16.19 \\
\hline & Smartwatch-assisted CCs vs. & 2Q vs. 1Q & 0.39 & 0.19 & 0.03 & 0.76 & 2.13 & & 1.49 & 1.03 & 2.14 \\
\hline & Non-smartwatch-assisted & $3 \mathrm{Q}$ vs. $1 \mathrm{Q}$ & 0.59 & 0.24 & 0.12 & 1.05 & 2.47 & \multirow[t]{2}{*}{0.015} & 1.80 & 1.13 & 2.87 \\
\hline & CCs & 4Q vs. 1Q & 0.77 & 0.25 & 0.28 & 1.26 & 3.11 & & 2.16 & 1.33 & 3.52 \\
\hline
\end{tabular}

TFT, two-finger technique; TTHT, two-thumb encircling hands technique; $Q$, quartile; $1 \mathrm{Q}$, first quartile; $2 \mathrm{Q}$, second quartile; $3 \mathrm{Q}$, third quartile; $4 \mathrm{Q}$, fourth quartile.

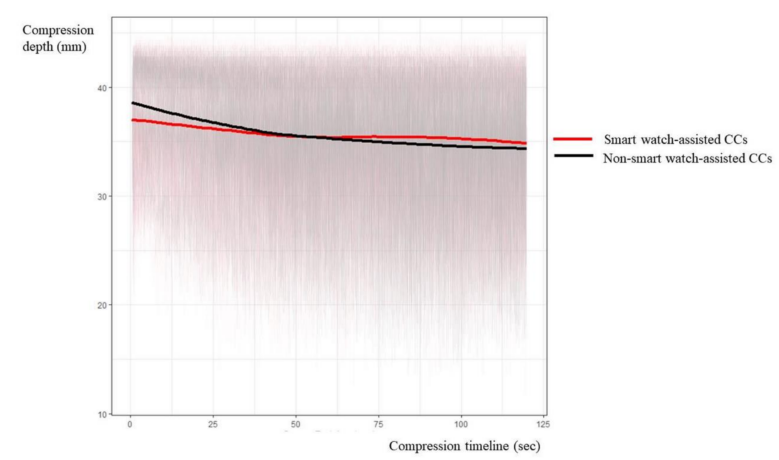

Figure 3. Comparison of the timeline graphs of compression depth between smart watch-assisted CCs and non-smartwatch-assisted CCs. CC, chest compression. 
In another subgroup analysis, the TTHT group achieved the adequate compression depth 8.5 times better than the TFT group ( $p$-Value < 0.001). The AOR was 8.49 (95\% CI, 4.45-16.19) for the TTHT in comparison with the TFT. The TTHT produced stronger and more consistent CCs than the TFT without the compromising the quality of compressions.

\section{Discussion}

This study showed that the haptic feedback system using a smartwatch improves the quality of infant CPR by maintaining proper speed and depth regardless of the compression methods. The proportion of optimal CC duration was higher and the absolute difference from the adequate compression rate was much smaller in the smartwatch-assisted group than in the non-smartwatch-assisted group. The participants were able to maintain the CCs at a constant duration over time when assisted by a smartwatch. Although the mean CC depth was not significantly different between the groups, subgroup analysis showed that the CC depth of the smartwatch-assisted group was appropriate over time.

The prompt feedback on the duration of CCs may result in an add-on effect of enhancing the CCs [22]. Previous studies have shown that inappropriate compression rates may lead to poor quality of compressions [23]. During cardiac compression, if the compression rate is misjudged, it may be difficult to maintain continuous CC rates, eventually leading to a decrease in compression depth [22]. The survival rate and neurological outcomes after infant CPR are closely related to the quality of resuscitation [2]. Hence, smartwatches can be used by medical professionals to enhance the efficiency of infant CPR.

Recently, the development of wearable electronic devices is attracting attention given the advances in health care. Furthermore, these devices can help overcome the differences in medical resource quality between low-income and high-income countries [24]. Previously, audio-visual feedback devices have been used during CPR, which were not effective in noisy environments, had spatial constraints, and were expensive $[12,14,16,25]$. However, now we can improve the quality of infant CPR with a handy smartwatch that is not limited by spatial constraints, is cost-effective, and is easy to use. Smartwatch feedback provides generally consistent CC duration and depth without being affected by the compression method.

Previous studies have been often been limited to adult CPR [21,26] because it is difficult to apply existing devices to infants and the incidence of infant CPR is low. The other factor is that in infant CPR, there are two types of CC methods involving the use of two fingers, whereas in adult CPR, both hands are used [12]. It has been reported that real-time feedback can improve the quality of CC even with increased fatigue during infant CPR [15]. We proved that smartwatch feedback provides generally consistent CC duration and depth during compression statistically. Therefore, the haptic feedback system using a smartwatch may help maintain high-quality CC despite increased fatigue.

While previous studies focused on medical students, our study involved medical professionals [17]. In fact, medical professionals engage more often in adult CPR than in infant CPR given its low incidence, so these haptic feedback devices could be helpful in clinical settings [1]. We can expect the smartwatch feedback to be effective when the educational effect declines [27].

We examined the effects of the use of smartwatch on the two controversial methods (TFT vs. TTHT) in our subgroup analysis. Previous studies have reported that chest compression using two fingers increases fatigue and reduces compression quality [28]. Between TFT and TTHT, the optimal CC duration and rate did not show a significant difference. However, the TTHT group achieved adequate compression depth about 8.5 times better than the TFT group $(p$-Value $<0.001)$. Thus, the TTHT provided stronger and more consistent CCs than the TFT without compromising the quality of compression.

This study has several limitations. First, this was a simulation study that used a mannequin, so the results have limited applicability to real clinical settings. We assumed that haptic feedback using vibrations from a smartwatch can be effective in noisy environments. However, in real clinical practice, there may be factors that cause interference with the 
compressors' performance in crowded environments [12]. Research with haptic devices at actual medical sites should be considered in future. Second, the intensity of the vibration from the smartwatch cannot be modified and varies according to producers. Participants may feel vibration intensity differently due to individual perceptions and CC motion [21]. If the vibration intensity is too weak or strong, it can affect the quality of compression. Therefore, further studies are required to optimize the intensity of vibration via smartwatches for individual users. Third, because the actual resuscitation situation is often noisy and urgent, it can affect the time to wear the smartwatch and operate the application, and these aspects were not assessed in this current study. Further studies are needed to identify factors that affect the time to operate the feedback system. Fourth, it would be less uncomfortable to wear a smartwatch during infant CPR due to two fingers being used, whereas both hands are used in adult CPR. However, depending on the individual, it may be uncomfortable to wear a smartwatch and perform chest compressions. Using a flexible and wearable form of haptic device or thin, soft rubber-band smartwatch can be an alternative to the inconvenience of wearing a hard smartwatch during CPR [29]. Therefore, further studies are required to assess how comfortable it is to individuals performing CPR with a smartwatch.

\section{Conclusions}

In conclusion, using a smartwatch as a feedback device ensures the delivery of highquality CPR to infants by medical professionals. In this simulation study, we showed that smartwatch feedback improves the adequacy of the CC duration and depth regardless of the compression method used.

Author Contributions: Conceptualization, S.A.J. and T.K.; Methodology, S.A.J. and T.K.; Validation, H.C., S.Y.Y., W.C.C. and T.K.; Formal Analysis, N.H. and K.K.; Data Curation, S.Y.H., H.Y. and T.K.; Writing-Original Draft Preparation, S.A.J. and T.K.; Writing-Review \& Editing, S.A.J. and T.K.; Visualization, S.A.J. and T.K.; Supervision, T.G.S., W.C.C. and T.K. All authors have read and agreed to the published version of the manuscript.

Funding: This research received no external funding.

Institutional Review Board Statement: This study was conducted according to the guidelines of the Declaration of Helsinki and approved by the Institutional Review Board of Samsung Medical Center (approval No. 2019-07-042-007/02.12.2019).

Informed Consent Statement: Informed consent was obtained from all subjects involved in the study. Written informed consent has been obtained from the patient(s) to publish this paper.

Data Availability Statement: The data for this study has been archived at the Department of Emergency Medicine, Samsung Medical Center, Sungkyunkwan University School of Medicine, Seoul, Korea. The data is private property of Samsung Medical Center, Sungkyunkwan University School of Medicine. The data is available on request as per the University Policy.

Conflicts of Interest: The authors declare no potential conflict of interests.

\section{References}

1. Atkins, D.L.; Everson-Stewart, S.; Sears, G.K.; Daya, M.; Osmond, M.H.; Warden, C.R.; Berg, R.A.; ROC Investigators. Epidemiology and outcomes from out-of-hospital cardiac arrest in children: The ROC Epistry-Cardiac Arrest. Circulation 2009, 119, 1484-1491.

2. Fink, E.L.; Prince, D.K.; Kaltman, J.R.; Atkins, D.L.; Austin, M.; Warden, C.; Hutchison, J.; Daya, M.; Goldberg, S.; Herren, H.; et al. Unchanged pediatric out-of-hospital cardiac arrest incidence and survival rates with regional variation in North America. Resuscitation 2016, 107, 121-128. [CrossRef]

3. Feneley, M.P.; Maier, G.W.; Kern, K.B.; Gaynor, J.W.; Gall, S.A.; Sanders, A.B.; Raessler, K.; Muhlbaier, L.H.; Rankin, J.S.; Ewy, G.A. Influence of compression rate on initial success of resuscitation and 24 hour survival after prolonged manual cardiopulmonary resuscitation in dogs. Circulation 1988, 77, 240-250. [CrossRef]

4. Meaney, P.A.; Bobrow, B.J.; Mancini, M.E.; Christenson, J.; de Caen, A.R.; Bhanji, F.; Abella, B.S.; Kleinman, M.E.; Edelson, D.P.; Berg, R.A.; et al. Cardiopulmonary resuscitation quality: [corrected] improving cardiac resuscitation outcomes both inside and outside the hospital: A consensus statement from the American Heart Association. Circulation 2013, 128, 417-435. [CrossRef] 
5. Maconochie, I.K.; Bingham, R.; Eich, C.; Lopez-Herce, J.; Rodriguez-Nunez, A.; Rajka, T.; Van de Voorde, P.; Zideman, D.A.; Biarent, D.; Paediatric Life Support Section, Collaborators. European Resuscitation Council Guidelines for Resuscitation 2015: Section 6. Paediatric life support. Resuscitation 2015, 95, 223-248. [PubMed]

6. Monsieurs, K.G.; Nolan, J.P.; Bossaert, L.L.; Greif, R.; Maconochie, I.K.; Nikolaou, N.I.; Perkins, G.D.; Soar, J.; Truhlar, A.; Wyllie, J.; et al. European Resuscitation Council Guidelines for Resuscitation 2015: Section 1. Executive summary. Resuscitation 2015, 95, 1-80. [CrossRef]

7. Atkins, D.L.; Berger, S.; Duff, J.P.; Gonzales, J.C.; Hunt, E.A.; Joyner, B.L.; Meaney, P.A.; Niles, D.E.; Samson, R.A.; Schexnayder, S.M. Part 11: Pediatric Basic Life Support and Cardiopulmonary Resuscitation Quality: 2015 American Heart Association Guidelines update for cardiopulmonary resuscitation and emergency cardiovascular care (reprint). Pediatrics 2015, 136, S167-S175. [CrossRef] [PubMed]

8. De Caen, A.R.; Berg, M.D.; Chameides, L.; Gooden, C.K.; Hickey, R.W.; Scott, H.F.; Sutton, R.M.; Tijssen, J.A.; Topjian, A.; Van Der Jagt, É.W.; et al. Part 12: Pediatric Advanced Life Support: 2015 American Heart Association guidelines update for cardiopulmonary resuscitation and emergency cardiovascular care (reprint). Pediatrics 2015, 136, S176-S195. [CrossRef]

9. Kleinman, M.E.; Brennan, E.E.; Goldberger, Z.D.; Swor, R.A.; Terry, M.; Bobrow, B.J.; Gazmuri, R.J.; Travers, A.H.; Rea, T. Part 5: Adult Basic Life Support and Cardiopulmonary Resuscitation Quality: 2015 American Heart Association guidelines update for cardiopulmonary resuscitation and emergency cardiovascular care. Circulation 2015, 132, S414-S435. [CrossRef]

10. Link, M.S.; Berkow, L.C.; Kudenchuk, P.J.; Halperin, H.R.; Hess, E.P.; Moitra, V.K.; Neumar, R.W.; O’Neil, B.J.; Paxton, J.H.; Silvers, S.M.; et al. Part 7: Adult Advanced Cardiovascular Life Support: 2015 American Heart Association guidelines update for cardiopulmonary resuscitation and emergency cardiovascular care. Circulation 2015, 132, S444-S464. [CrossRef] [PubMed]

11. Perkins, G.D.; Olasveengen, T.M.; Maconochie, I.; Soar, J.; Wyllie, J.; Greif, R.; Lockey, A.; Semeraro, F.; Van De Voorde, P.; Lott, C.; et al. European Resuscitation Council guidelines for resuscitation: 2017 update. Resuscitation 2018, 123, 43-50. [CrossRef] [PubMed]

12. Kim, C.W.; Oh, J.H. Effect of metronome guidance on infant cardiopulmonary resuscitation. Eur. J. Pediatr. 2019, 178, 795-801. [CrossRef]

13. Yu, B.G.; Oh, J.H.; Kim, Y.; Kim, T.W. Accurate measurement of chest compression depth using impulse-radio ultra-wideband sensor on a mattress. PLoS ONE 2017, 12, e0183971. [CrossRef]

14. Kirkbright, S.; Finn, J.; Tohira, H.; Bremner, A.; Jacobs, I.; Celenza, A. Audiovisual feedback device use by health care professionals during CPR: A systematic review and meta-analysis of randomised and non-randomised trials. Resuscitation 2014, 85, 460-471. [CrossRef]

15. Martin, P.; Theobald, P.; Kemp, A.; Maguire, S.; Maconochie, I.; Jones, M. Real-time feedback can improve infant manikin cardiopulmonary resuscitation by up to 79\%-A randomised controlled trial. Resuscitation 2013, 84, 1125-1130. [CrossRef] [PubMed]

16. Abella, B.S.; Edelson, D.P.; Kim, S.; Retzer, E.; Myklebust, H.; Barry, A.M.; O’Hearn, N.; Hoek, T.L.V.; Becker, L.B. CPR quality improvement during in-hospital cardiac arrest using a real-time audiovisual feedback system. Resusciation 2007, 73, 54-61. [CrossRef]

17. Lee, J.; Song, Y.; Oh, J.; Chee, Y.; Ahn, C.; Shin, H.; Kang, H.; Lim, T.H. Smartwatch feedback device for high-quality chest compressions by a single rescuer during infant cardiac arrest: A randomized, controlled simulation study. Eur. J. Emerg. Med. 2019, 26, 266-271. [CrossRef] [PubMed]

18. Song, Y.; Chee, Y.; Oh, J.; Ahn, C.; Lim, T.H. Smartwatches as chest compression feedback devices: A feasibility study. Resuscitation 2016, 103, 20-23. [CrossRef] [PubMed]

19. Reeder, B.; David, A. Health at hand: A systematic review of smart watch uses for health and wellness. J. Biomed. Inform. 2016, 63, 269-276. [CrossRef]

20. Lu, T.-C.; Chang, Y.-T.; Ho, T.-W.; Chen, Y.; Lee, Y.-T.; Wang, Y.-S.; Chen, Y.-P.; Tsai, C.-L.; Ma, M.H.-M.; Fang, C.-C.; et al. Using a smartwatch with real-time feedback improves the delivery of high-quality cardiopulmonary resuscitation by healthcare professionals. Resuscitation 2019, 140, 16-22. [CrossRef]

21. Ahn, C.; Lee, J.; Oh, J.; Song, Y.; Chee, Y.; Lim, T.H.; Kang, H.; Shin, H. Effectiveness of feedback with a smartwatch for high-quality chest compressions during adult cardiac arrest: A randomized controlled simulation study. PLoS ONE 2017, 12, e0169046. [CrossRef]

22. Lin, C.-Y.; Hsia, S.-H.; Lee, E.-P.; Chan, O.-W.; Lin, J.-J.; Wu, H.-P. Effect of audiovisual cardiopulmonary resuscitation feedback device on improving chest compression quality. Sci. Rep. 2020, 10, 398. [CrossRef] [PubMed]

23. Monsieurs, K.G.; De Regge, M.; Vansteelandt, K.; De Smet, J.; Annaert, E.; LeMoyne, S.; Kalmar, A.F.; Calle, P.A. Excessive chest compression rate is associated with insufficient compression depth in prehospital cardiac arrest. Resuscitation 2012, 83, 1319-1323. [CrossRef]

24. Lu, L.; Zhang, J.; Xie, Y.; Gao, F.; Xu, S.; Wu, X.; Ye, Z. Wearable health devices in health care: Narrative systematic review. JMIR mHealth uHealth 2020, 8, e18907. [CrossRef] [PubMed]

25. Wee, J.C.P.; Nandakumar, M.; Chan, Y.H.; Yeo, R.S.L.; Kaur, K.; Anantharaman, V.; Yap, S.; Ong, M.E.H. Effect of using an audiovisual CPR feedback device on chest compression rate and depth. Ann. Acad. Med. Singap. 2014, 43, 33-38. 
26. Choi, B.; Kim, T.; Yoon, S.Y.; Yoo, J.S.; Won, H.-J.; Kim, K.; Kang, E.J.; Yoon, H.; Hwang, S.Y.; Shin, T.G.; et al. Effect of watch-type haptic metronome on the quality of cardiopulmonary resuscitation: A simulation study. Health Inform. Res. 2019, 25, $274-282$. [CrossRef]

27. Berden, H.J.; Willems, F.F.; Hendrick, J.M.; Pijls, N.H.; Knape, J.T. How frequently should basic cardiopulmonary resuscitation training be repeated to maintain adequate skills? BMJ 1993, 306, 1576-1577. [CrossRef]

28. Haque, I.U.; Udassi, J.P.; Udassi, S.; Theriaque, U.W.; Shuster, J.J.; Zaritsky, A.L. Chest compression quality and rescuer fatigue with increased compression to ventilation ratio during single rescuer pediatric CPR. Resuscitation 2008, 79, 82-89. [CrossRef]

29. Pece, F.; Zarate, J.J.; Vechev, V.; Besse, N.; Gudozhnik, O.; Shea, H.; Hilliges, O. MagTics: Flexible and Thin Form Factor Magnetic Actuators for Dynamic and Wearable Haptic Feedback. In Proceedings of the 30th Annual ACM Symposium on User Interface Software and Technology, Quebec, QC, Canada, 22-25 October; 2017; pp. 143-154. 